\title{
The relationship between child anxiety and the quality of life of children, and parents of
}

children, on the autism spectrum.

Dawn Adams ${ }^{1,2}$, Megan Clark $^{1,2}$, Kate Simpson ${ }^{1,2}$

${ }^{1}$ Autism Centre of Excellence, School of Education and Professional Studies, Griffith University, Brisbane, Australia

${ }^{2}$ Griffith Institute of Educational Research, Griffith University, Brisbane, Australia

Running Head: Anxiety and quality of life

Correspondence regarding this article should be addressed to:

Dr Dawn Adams

Autism Centre of Excellence

School of Education and Professional Studies

Griffith University

Mount Gravatt, Brisbane

QLD 4122

Australia

Email: dawn.adams@griffith.edu.au

\section{Acknowledgements}

We are grateful to the children with autism and their families for giving their time to support this research study.

Funding statement and declaration of conflicting interests

Dawn Adams declares that she has no conflict of interest with respect to this publication.

Megan Clark declares that she has no conflict of interest with respect to this publication.

Kate Simpson declares that she has no conflict of interest with respect to this publication. 
The authors acknowledge the financial support of an internal Griffith University Education and Professional Studies internship for assistance with data entry. 
The relationship between child anxiety and quality of life in children, and parents of children, on the autism spectrum.

Children on the autism spectrum experience high rates of anxiety but little is known about the impact of anxiety on child or parent quality of life (QoL). This study aimed to investigate the relationship between anxiety, autism characteristics, and QoL in children and their parents. Sixty-four parents of children on the spectrum completed questionnaires on their child's autism characteristics, anxiety symptomatology, and both child (PedsQL) and parent QoL (WHOQoL-BREF). Parents of children with elevated anxiety reported lower child and parent QoL. Regression models highlight specific anxiety subscales as predictive of PedQL school and emotional functioning but not of parent QoL. Anxiety symptomatology may be a significant factor contributing to specific aspects of QoL for children on the spectrum.

Keywords: autism, anxiety, health-related quality of life 
The autism spectrum is a lifelong neurodevelopmental condition with a current prevalence rate of 1 in 59 individuals (CDC, 2018). Children on the autism spectrum are more vulnerable to anxiety than are typically developing children, with up to $40 \%$ of children on the autism spectrum meeting criteria for a co-morbid anxiety disorder (van Steensel, Bogels, \& Perrin, 2011). Moreover, it is not uncommon for individuals on the autism spectrum to concurrently experience more than one type of anxiety (Rodgers et al., 2017). The presence of an anxiety disorder has been found to cause functional impairment over and above the characteristics of autism (Matson \& Nebel-Schwalm, 2007). Despite anxiety symptoms being amongst the most common presenting concerns for children and adolescents on the spectrum (White et al., 2015), both clinicians and researchers can find it inherently difficult to assess anxiety symptoms in individuals on the spectrum due (at least in part) to the overlapping symptomatology of the two conditions that increases the challenge of delineating autism and anxiety (Rodgers et al., 2016).

There is increasing evidence that whilst some aspects of anxiety are similar between those with and without a diagnosis of autism, there are also some aspects of anxiety which can manifest differently in children on the spectrum. The presentation of anxiety can vary as a function of autism characteristics or profile, associated autism diagnostic subtype, age of the child, cognition and language abilities (Caporino et al., 2017; Lecavalier et al., 2014; Wigham, Rodgers, South, McConachie, \& Freeston, 2015) and/or environment (Adams, Young, Simpson, \& Keen, 2018). There is also the suggestion of atypical as well as typical signs of anxiety (Kerns \& Kendall, 2012) as well as an increased prevalence of difficulties with tolerating uncertainty (den Houting, Adams, Roberts, \& Keen, 2018b). The potential differences in anxiety symptomatology combined with the potential for overlap with autism characteristics have led to growing concern around the validity and reliability of assessing anxiety symptomatology in individuals on the autism spectrum using instruments originally 
developed to capture anxiety in typically developing children. To that end, the Anxiety Scale for Children with ASD (ASC-ASD; Rodgers et al. 2016) was adapted from the Revised Child Anxiety and Depression Scale (Chorpita, Yim, Moffitt, Umemoto, \& Francis, 2000) to include items that measure anxiety around sensory experiences, difficulties with uncertainty, and specific phobias, bridging the gap in the assessment of anxiety in children on the spectrum. Adapting a measure of anxiety designed for typically developing children allowed for the recognition of the overlap of anxiety experiences between those with and without a diagnosis on the spectrum as well as measuring additional autism-specific factors not captured on measures designed for typically developing individuals.

While the high prevalence of anxiety in the autism population has been recognised, the impact of anxiety on the functioning of children on the autism spectrum is not well understood. To date, there has been a greater focus on how anxiety impacts upon the development, health, and well-being of typically developing children. Childhood anxiety has been found to implicate the development of typically developing children in a number of ways. Children experiencing anxiety are more vulnerable to cognitive and language impairments and psychopathology such as depression later in life (Beesdo, Knappe, \& Pine, 2009). Further, anxiety in typically developing children has been associated with increased social competence deficits and, thus, has been associated with poorer social outcomes when compared to children without anxiety (Spence, Donovan, \& Brechman-Toussaint, 1999). Sleep disturbances associated with childhood anxiety are also well documented (Caporino et al., 2017; Gregory et al., 2011; Gregory \& Sadeh, 2012; Ivanenko, McLaughlin Crabtree, O'Brien \& Gozal, 2006) and can heighten a child's risk of other health complications (Mahrer, Montano, \& Gold, 2012). To date, research has not extensively explored the impact of anxiety in children on the autism spectrum, so it is not yet known if patterns are similar to or different from those noted in typically developing children. While the functional 
impairment varies depending on the nature and severity of the specific anxiety condition (Beesdo et al., 2009), findings from typically developing children suggest that childhood anxiety can have negative repercussions for a child's quality of life.

\section{Quality of life in children on the autism spectrum}

Quality of life (QoL) is a broad, multidimensional construct defined by the World Health Organisation (WHO) Group as the subjective perception of one's life and well-being, assessing both positive and negative dimensions of functioning (The WHOQOL Group, 1998). Health-related quality of life (HRQoL) is a subjective and multidimensional construct under the QoL umbrella that focuses on the dimensions of QoL more directly related to physical or mental health and includes the domains related to physical activities, positive health behaviours, school functioning, and psychosocial well-being. It is therefore well suited to conditions that have a multidimensional impact, such as the autism spectrum (Kuhlthau et al., 2010).

Children and youth with a diagnosis on the autism spectrum are frequently reported to have poorer QoL and specifically, poorer HRQoL when compared with their typically developing peers (Kuhlthau et al., 2010; Lee, Harrington, Louie, \& Newschaffer, 2008; Limbers, Heffer, \& Varni, 2009; Sikora, Vora, Coury, \& Rosenberg, 2012; van Heijst \& Geurts, 2015) and this continues across the lifespan (van Heijst \& Geurts, 2015). They are also reported to have overall poorer HRQoL than children with psychiatric disorders (Bastiaansen, Koot, Bongers, Varni, \& Verhulst, 2004). HRQoL is an important measure of outcome for individuals on the autism spectrum as it provides a comprehensive view of functioning across multidimensions including physical, social, emotional, and environmental functioning. Bastiaansen et al. (2004) highlighted the importance of considering HRQoL at this domain level as differences are reported across the HRQoL domains across disorders. For example, based on parent reporting, children and youth on the autism spectrum score 
within the normative range on the domains of autonomy and parent relations (Biggs \& Carter, 2016; Egilson, Olafsdottir, Leosdottir, \& Saemundsen, 2017) and school environment (Biggs \& Carter, 2016). By contrast, scores on the social functioning (Egilson et al., 2017), emotional functioning (Bastiaansen et al., 2004), and psychological health subdomains (Tung et al., 2014) are often reportedly lower. Exploration of specific domains, and the predictors of these specific domains, therefore allows for a more accurate description of HRQoL within children on the autism spectrum, which is critical should targeted interventions be developed.

\section{Predictors of HRQoL in autism}

In an attempt to better understand HRQoL in autism and ways of providing support for children on the autism spectrum, research has moved towards identifying the factors that are predictive of HRQoL outcomes. Chiang and Wineman (2014) reviewed 16 studies of HRQoL within autism to identify the factors associated with increased HRQoL. While the influence of autism characteristics and adaptive behaviour on HRQoL outcomes has yielded mixed results in the literature, more behaviour problems, greater social impairment, and poorer social communication skills were identified as common predictors of HRQoL in both adults and children. Intellectual ability has also been associated with HRQoL outcomes of children and youth on the autism spectrum, where co-occurring intellectual disability (ID) is often related to poorer HRQoL outcomes than for children on the autism spectrum without ID (Arias et al., 2018; Chiang \& Wineman, 2014; Totsika, Felce, Kerr, \& Hastings, 2010). These results identify that factors other than, or in addition to, characteristics of autism predict HRQoL.

While anxiety has been associated with increased psychological and physical health concerns (Bittner et al., 2007), social difficulties (Gregory \& Sadeh, 2012; Kingery, Erdley, Marshall, Whitaker, \& Reuter, 2010), and sleep disturbances (Caporino et al., 2017; Gregory 
et al., 2011), and poorer HRQoL, in typically developing children (Gilbertson, Morgan, Rapee, Lyneham, \& Bayer, 2017), we cannot assume that anxiety will impact children on the autism spectrum in the same way. Similarly, we cannot assume that a child on the autism spectrum will have poorer HRQoL due to their diagnosis alone, without properly understanding other potential factors contributing to their HRQoL. According to Tavernor, Barron, Rodgers, and McConachie (2013), anxiety may be a key factor which impacts upon the HRQoL of children on the autism spectrum, highlighting the need for studies which explore this relationship in detail. Steensel, Bogels, and Dirksen (2012) explored the impact of anxiety and the impact of autism characteristics on QoL in two separate analyses, with the results highlighting a stronger parameter estimate of autism characteristics than anxiety symptoms, but as both autism and anxiety were not entered into a single analysis, the relative contribution of each factor in the presence of the other factor cannot be deduced. This substantiates the importance of further exploring how psychiatric conditions such as anxiety can impact the HRQoL of children on the autism spectrum.

Compared with other paediatric conditions, there has been less focus on the HRQoL of children on the autism spectrum (Tavernor et al., 2013). While the research on HRQoL in children and adolescents on the autism spectrum is continuing to emerge, there is limited knowledge about the factors associated with HRQoL in children on the autism spectrum and additional research is needed to firmly establish the relative contribution of anxiety symptomatology and autism characteristics on the HRQoL. Understanding the impact of anxiety on the physical, social, emotional, and psychological functioning domains of children on the autism spectrum can help to inform appropriate provisions during childhood to minimize maladjustment and to improve well-being during adolescence and adulthood. 
Although research in typically developing children has established associations between child emotional and behavioural problems, including anxiety, and elements of parental wellbeing (e.g. Crnic et al., 2005; Ford et al., 2004), there has been limited research exploring this relationship within children on the autism spectrum. A recent systematic review into QoL in parents of children on the autism spectrum showed that all studies reported lower QoL in parents of children with autism than in parents of typically developing children in at least one domain (Vasilopoulou \& Nisbet, 2016). Four of their identified studies explored the impact of emotional problems on parental Qol, with only two studies reporting a significant correlation between either child emotional symptoms (Totsika, Hastings, Emerson, Berridge \& Lancaster, 2011) or child emotional QoL (Bourke-Taylor, Pallant, Law \& Howie, 2012) and parental QoL. No studies explored the impact of specific diagnoses, such as anxiety, and parental QoL. There were also mixed results within the studies included in the review in relation to child autism characteristics or severity and parental QoL. Such mixed results led Vasilopoulou \& Nisbet (2016) to conclude that further exploration of parental QoL (with a broad instrument such as the WHOQoL-Bref) and its predictors is required.

Aims

The current study aimed to investigate the individual contribution of specific aspects of anxiety symptomatology and autism characteristics in the prediction of child HRQoL in a sample of school-aged children on the autism spectrum. The current study extends existing work in the field of child QoL which have only considered the total score on HRQoL measures by exploring both the total score of the Pediatric Quality of Life (PedsQL) scale and the four subdomains (physical, social, emotional, and school functioning) to assess for subscale differences. The study will also be the first to use the autism-specific measure of 
anxiety (ASC-ASD) as a predictor of HRQoL, exploring the impact of overall anxiety as well as the relative contribution of the specific subscales.

In addition to this, given the established relationships between child anxiety and parental QoL in typically developing children and the highlighted need for further work exploring correlates and predictors of parental QoL in parents of children with autism (Vasilopoulou \& Nisbet, 2016), this study will explore the impact of child anxiety on parental QoL at both the total score and subscale level. The study will also be the first to use the autism-specific measure of anxiety (ASC-ASD) as a predictor of parental QoL, exploring the impact of overall anxiety as well as the relative contribution of the specific subscales.

Based on the previous literature, the following hypotheses were made:

\section{Hypotheses}

1. Autism characteristics have previously been shown to be predictive of a child's HRQoL over and above a child's age and ability (de Vries \& Geurts, 2015). Therefore, it was hypothesised that a child's autism characteristics (as measured by the Social Communication Questionnaire [SCQ]) would significantly predict child HRQoL (measured using the PedsQL) as reported by their parents.

2. It has also been established that co-occurring mental health diagnoses including anxiety were predictive of poorer child HRQoL (Kose et al., 2013; Sikora et al., 2012). Therefore, it was expected that anxiety symptomatology (measured by the ASC-ASD) would predict the HRQoL of school-aged children on the autism spectrum.

3. Given the previous work by van Steensel et al. (2012), it was hypothesised that autism characteristics would be a stronger predictor of HRQoL than would anxiety symptoms. 
Given the mixed and conflicting findings in the systematic review by Vasilopoulou \& Nisbet (2016) in relation to predictors of parental QoL, no hypotheses were made about the impact of anxiety and autism characteristics on parental QoL.

\section{Methods}

Ethical clearance for this study was granted through the $<$ removed for blind review $>$ University Human Research Ethics Committee, approval number xxx/xxx.

\section{Recruitment procedures}

Parents of children on the autism spectrum were recruited through social media posts and parent information sessions held at the University. Parents could complete the questionnaire online or using pen and paper. In total, 91 parents completed and returned the

questionnaire. Twelve were excluded as they had a total score on the Social Communication Questionnaire (SCQ) (Rutter, Bailey, Lord \& Berument, 2003) below the cut-off of 15 or their SCQ questionnaire was incomplete. A further 15 were excluded as the child was below the age of 5 or the child's age was not provided.

\section{Participants}

After exclusions, the sample consisted of data on 64 children on the autism spectrum. The sample was predominantly male (71.9\%) with a mean age of 10 years 1 month $(S D 3$ years 1 month), ranging from 5-16 years. Based upon parent-report, children had a range of levels of self-help and adaptive skills. All children were reported to be continent all of the time or with only occasional incidents for both wetting and soiling during the day. Almost all $(92.2 \%)$ were fully continent for soiling during the night with the remaining five children (7.8\%) reported to have occasional night-time soiling incidents. Nine $(14.1 \%)$ children were reported to have frequent wetting incidents at night, seven $(10.9 \%)$ occasional night-time 
wetting incidents; the remaining $75 \%(n=48)$ of the sample were reported to be continent (wetting) at night. When asked about their child's washing and dressing skills, $67.2 \%$ of parents said that their child is able to wash and dress independently, $28.1 \%$ were able to wash with help and $29.7 \%$ were able to dress with help. Three children (aged 5.3 years, 6.25 years and 11.5 years) were unable to wash themselves even with help and two children (aged 5.3 years and 11.5 years) were unable to dress themselves with help. A small group had moderate to significant hearing difficulties $(n=6,9.9 \%)$ vision difficulties $(n=5,8.2 \%)$ or delayed speech $(n=5,7.9 \%)$. Twenty-four $(37.5 \%)$ children had a parent-reported diagnosis of anxiety (which is concordant with estimates from meta-analyses, e.g., van Steensel et al., 2011), of whom six were reported to be prescribed selective serotonin reuptake inhibitors (SSRIs). Twenty-one (32.8\%) children were reported to have a diagnosis of an attention deficit disorder, of whom nine were prescribed Methylphenidate.

Parent informants were mostly mothers (96.9\%) and no respondents reported living separately from the child. Six (9.4\%) parents reported having a diagnosis of an autism spectrum disorder and 17 (26.6\%) reported having a diagnosis of a mental health difficulty. The majority of the respondents reported having at least a tertiary education $(89.1 \%)$ and having a family income in excess of AUD \$80,000 (67.2\%). For reference, the 2016 Australian Census reported the median Australian annual family income to be AUD \$90,168.

\section{Measures}

Demographic characteristics. These were collected using a parent questionnaire focusing upon child (age, gender, age at diagnosis, self-help skills, hearing, speech or language skills), parent (education, employment), and household (income) variables.

Autism characteristics. The SCQ (Rutter et al., 2003) is a behavioural checklist that requires parents to indicate the presence of certain social, communicative, or stereotyped behaviours by answering yes or no to 40 items. The SCQ has been extensively researched, 
with a recent meta-analysis (Chesnut, Wei, Barnard-Brak, \& Richman, 2017) concluding that it is an acceptable screening measure for autism spectrum disorder (area under the curve .89) if used within the correct age range. A higher score represents a higher number of behaviours which may be considered indicative of autism.

Child anxiety. The Anxiety Scale for Children - Autism Spectrum Disorder Parent Form (ASC-ASD-P) (Rodgers et al., 2017) consists of 24 items which divide into four subscales: Performance Anxiety (five items; maximum score 15); Anxious Arousal (six items; maximum score 18); Separation Anxiety (five items; maximum score 15), and Uncertainty (eight items; maximum score 24). Severity was rated on a 4-point scale ranging from 0 (never) to 3 (always). Although the scale was originally developed for children aged 8 or above, it has been used in younger samples of children on the autism spectrum to describe anxiety symptomatology (Keen, Adams, Simpson, den Houting, \& Roberts, 2017). The scale has good validity, reliability, and internal consistency and is highly correlated (suggesting good concurrent validity) with the Screen for Child Anxiety Related Emotional Disorders (SCARED) (Birmaher et al., 1999) and the Spence Child Anxiety Scale (SCAS; den Houting, Adams, Roberts \& Keen, 2018a), two robust measures of anxiety in the general population. den Houting et al. (2018a) and Keen et al. (2017) also report good discriminant validity against measures of autism characteristics. Adams, Simpson, and Keen (2018) explored PRMSE values for each subscale, concluding that this measure is statistically valid at subscale level and that subscales add clinical utility above using the total score alone. In the current study, internal consistency was identified as acceptable to excellent for all subscales based on Cronbach's alpha: Anxious Arousal $\alpha=.86$, Separation Anxiety $\alpha=.84$, Performance Anxiety $\alpha=.87$, and Uncertainty $\alpha=.86$.

Two indicative cut-off points for the ASC-ASD-P total scale have been put forward by the authors: scores $\geq 20$ suggest "significant anxious symptomatology", and scores $>24$ 
are considered to be a "more specific indication of significant anxiety" (Rodgers et al., n.d.). These cut-off scores have been explored in relation to an established measure of anxiety (Spence Child Anxiety Scale) and parent-reported clinical anxiety diagnosis status with $100 \%$ of children with a clinical anxiety diagnosis achieving a score of 20 or higher on the ASC-ASD-P and $71 \%$ of children with a clinical anxiety diagnosis achieving a score of 24 or higher (den Houting et al., 2018a). Within this study, the cut-off score of 24 will be used to indicate elevated anxiety symptomatology. The significant majority $(83.3 \%)$ of children within this sample who had a clinical diagnosis of anxiety achieved a score of 24 or more.

Child-health-related quality of life (C-HRQoL). The Pediatric Quality of Life Inventory 4.0 (PedsQL; Varni, Seid \& Kurtin, 2001) is one of the most widely studied and cited assessments of c-HRQoL. Informants respond to 23 items which cover the core health dimensions identified by the WHO. The informant is not asked to rate the presence or absence of a certain behaviour, but asked to rate how often their child has had problems with a specific task or experience. Four subscales can be derived: physical functioning (e.g., energy, activity, strength), emotional functioning (e.g., feeling angry, sleep, worry), social functioning (e.g., friendships, getting teased) and school functioning (e.g., attendance, completing schoolwork). The Psychosocial Health domain score consists of items within the emotional, social, and school functioning subscales. A higher score indicates a higher level of health-related quality of life. As the number of items differs across subscales, the mean score per question will be presented when describing subscale data. The PedsQL has been widely used in children on the autism spectrum (Kuhlthau et al., 2010; Thomas, Sciberras, Lycett, Papadopolous, \& Rinehart, 2018) and has been identified as having adequate reliability within this population (Stokes, Kornienko, Scheeren, Koot, \& Begeer, 2017). In the current study, internal consistency was identified as acceptable to excellent for all 
subscales based on Cronbach's alpha: Physical $\alpha=.85$, Emotional $\alpha=.74$, Social $\alpha=.76$ and School $\alpha=.76$.

Parent Quality of Life (P-QoL). The World Health Organization Quality of Life Assessment-BREF (WHOQOL-BREF) is an abbreviated form of the WHOQO1-100 and measures individuals' physical, psychological, social, and environmental health. It has been used to assess parental QoL in a number of studies of parents of children with autism (e.g. Dardas \& Ahmad, 2014; Tung, Huang, Tseng, Yen \& Tsai, 2014). The 26 items of the scale range from 1 to 5 with higher scores denoting higher QoL. As the number of items differs for each subscale, the mean score per question will be presented when describing subscale data. Both the subscale and the total QoL scores were used in the analyses. In the current study, internal consistency was identified as acceptable to excellent for all subscales based on Cronbach's alpha: Physical $\alpha=.81$, Emotional $\alpha=.87$, Social $\alpha=.70$ and Environmental $\alpha=.84$.

Data analysis

Data were screened to ensure they met assumptions of normality. First, c-HRQoL and p-QoL were compared between those who were above and below the cut-off indicative of anxiety on the ASC-ASD-P using one-way ANOVAs. Second, to study whether specific subscales of the ASC-ASD-P were predictors for different aspects of c-HRQoL and p-QoL, linear regression analyses were conducted with c-HRQoL and p-QoL subscales as the dependent variables and ASC-ASD-P subscale scores as predictor variables. Alpha was set to .05 for all analyses due to the exploratory nature of the study. Effect sizes were calculated using partial eta squared, where 0.01 is considered a small effect size, 0.09 a medium effect size and .25 a large effect size. Prior to examining prediction models, bivariate and pointserial correlation analyses were conducted. These revealed no collinearity of predictors $(r>.80)$, thus all were retained (see Table 1$)$. 
Table 1.

Pearson Correlations between Predictor Variables

\begin{tabular}{lcccc}
\hline & 2 & 3 & 4 & 5 \\
& & & & \\
\hline 1. SCQ total score & -.09 & -.08 & --.06 & .06 \\
2. ASC-ASD-P Performance & - & $.66^{* *}$ & $.63^{* *}$ & $.63^{* *}$ \\
3. ASC-ASD-P Anxious Arousal & & - & $.68^{* *}$ & $.69^{* *}$ \\
4. ASC-ASD-P Separation & & & - & $.62^{* *}$ \\
5. ASC-ASD-P Uncertainty & & & & - \\
\hline
\end{tabular}

$* p<.01 ; * * p<.001$.

\section{Results}

Profile of results on the ASC-ASD

Across the sample, ratings were given between 0 and 3 for each item on the ASCASD-P. The three items with the highest mean score (representing elevated anxiety) across the sample were "My child always needs to be prepared before things happen" (mean $=2.02$, $\mathrm{SD}=.95)$, "My child worries if they don't know what will happen next e.g. if plans change" $($ mean $=1.72, \mathrm{SD}=.97)$, and "My child is afraid of entering a room full of people" (mean $=$ $1.48, \mathrm{SD}=.94)$, all of which are from the Uncertainty subscale. The three items with the lowest mean score across the sample were all from the Anxious Arousal subscale: "My child suddenly becomes dizzy or faint when there is no reason for this" $($ mean $=0.28, \mathrm{SD}=.65)$, "My child suddenly starts to tremble or shake when there is no reason for this" $($ mean $=0.34$, $\mathrm{SD}=.70$ ), and "My child suddenly feels so anxious he/she feels as if he/she can't breathe when there is no reason for this" (mean $=0.47, \mathrm{SD}=.78)$.

The average total ASC-ASD-P was $25.9(\mathrm{SD}=14.6)$ which equates to a mean score per question of $1.1(\mathrm{SD}=0.65)$. Total ASC-ASD-P scores ranged from 3-70; $34(53 \%)$ of 
parent ratings placed their child in the range for "elevated anxiety". The subscale averages (mean score per question) were 1.2 $(\mathrm{SD}=0.8)$ for Performance Anxiety, $0.65(\mathrm{SD}=0.62)$ for Anxious Arousal, $1.0(\mathrm{SD}=0.89)$ for Separation Anxiety and $1.33(\mathrm{SD}=0.70)$ for Uncertainty.

Profile of results on the measure of c-HRQoL: PedsQL

Across the sample, ratings were given between 0 and 100 for each item on the PedsQL. The items with the highest mean scores (representing higher c-HRQoL and/or less difficulties in undertaking that activity) across the sample were predominantly from the Physical Functioning subscale; Walking more than a block (mean =77.7, $\mathrm{SD}=30.6)$, Running (mean $=69.5, \mathrm{SD}=34.4)$, Lifting something heavy $($ mean $=69.1, \mathrm{SD}=31.4)$. One item from the School Functioning subscale was the joint third highest rated item: Missing school to go to the doctor or hospital $($ mean $=69.1, \mathrm{SD}=26.6)$. The three items with the lowest mean average scores across the sample were Forgetting things (mean $=33.2, \mathrm{SD}=26.4)$, Keeping up with school activities/schoolwork $($ mean $=37.1, \mathrm{SD}=28.5)$ (both from the School Functioning domain) and Feeling angry (mean $=37.9, \mathrm{SD}=23.1)$.

The average total PedsQL score (mean score per question) was $50.8(\mathrm{SD}=15.6)$, with scores ranging from 22-95. The subscale averages (mean score per question) were 62.0 $(\mathrm{SD}=23.2)$ for Physical Functioning, $48.45(\mathrm{SD}=20.0)$ for Social Functioning, 47.7 (SD $=19.9)$ for School Functioning and $45.1(\mathrm{SD}=21.0)$ for Emotional Functioning.

Profile of results on the measure of p-QoL: WHOQOL-BREF

Across the sample, ratings were given between 1 and 5 for each item on the WHOQOLBREF with the exception of the questions which ask about having the information available needed in day-to-day life (item 13), how able they are to get around (item 15) and how satisfied respondents are with their access to health care services (question 24), all of which 
had a minimum score of 2 and maximum of 5 . Two of the three items with the highest mean scores (representing higher p-QoL and/or less difficulties in undertaking that activity) were from the Physical domain: How well are you able to get around? (mean = 4.6, $\mathrm{SD}=$ 0.71), How much do you need any medical treatment to function in your daily life? (mean = 4.1, $\mathrm{SD}=1.0$ ) and one was from the Environment domain: How satisfied are you with your transport? $($ mean $=4.4, \mathrm{SD}=0.72)$. Two of the three items with the lowest mean scores were from the Social domain; To what extent do you have the opportunity for leisure activities? (mean $=2.5, \mathrm{SD}=1.2)$, How satisfied are you with the support you get from your friends? $($ mean $=3.0, \mathrm{SD}=1.0)$ and one was from the Physical domain; How satisfied are you with your sleep? $($ mean $=3.0, \mathrm{SD}=1.1)$.

The average total WHO-QOL BREF score (mean score per question) was $3.6(\mathrm{SD}=$ 0.6), with scores ranging from 47-118. The domain averages (mean score per question), from highest to lowest were Environment $($ mean $=4.2, \mathrm{SD}=0.8)$, Physical (mean $=3.7, \mathrm{SD}$ $=0.7)$, Psychological (mean $=3.3, \mathrm{SD}=0.8)$ and Social $($ mean $=3.2, \mathrm{SD}=0.8)$.

\section{C-HRQoL in those with and without elevated anxiety symptomatology}

As summarised in Table 2, parents of children experiencing elevated anxiety symptomatology reported lower overall c-HRQoL scores for their children as well as significantly lower scores in the c-HRQoL physical, emotional, and school functioning subscales. All significant comparisons had a medium to large effect size with the exception of school functioning, which had a small to medium effect size.

\section{P-QoL in those with and without elevated anxiety symptomatology}

As summarised in Table 2, parents of children experiencing elevated anxiety symptomatology reported lower overall p-QoL scores (with a medium effect size) as well as 
significantly lower scores in the physical and psychological domains (with medium effect sizes) subscales.

C-HRQoL and P-QoL dependent upon child and parent demographic variables

Exploratory comparisons of c-HRQoL and p-QoL split by child gender, child age (median split) and parent mental health are documented in the supplementary material.

There were no significant differences in c-HRQoL and p-QoL based upon child gender or age. There were no significant differences in c-HRQoL based upon parent mental health diagnosis. Parents who reported a diagnosis of a mental health condition also reported lower physical, psychological, environmental and total p-QoL. 


\section{Table 2.}

PedsQL and WHOQoL-BREF Scores (Mean Score Per Question) split by Children Scoring Above and Below the ASC-ASD-P Cut-off for Elevated Anxiety

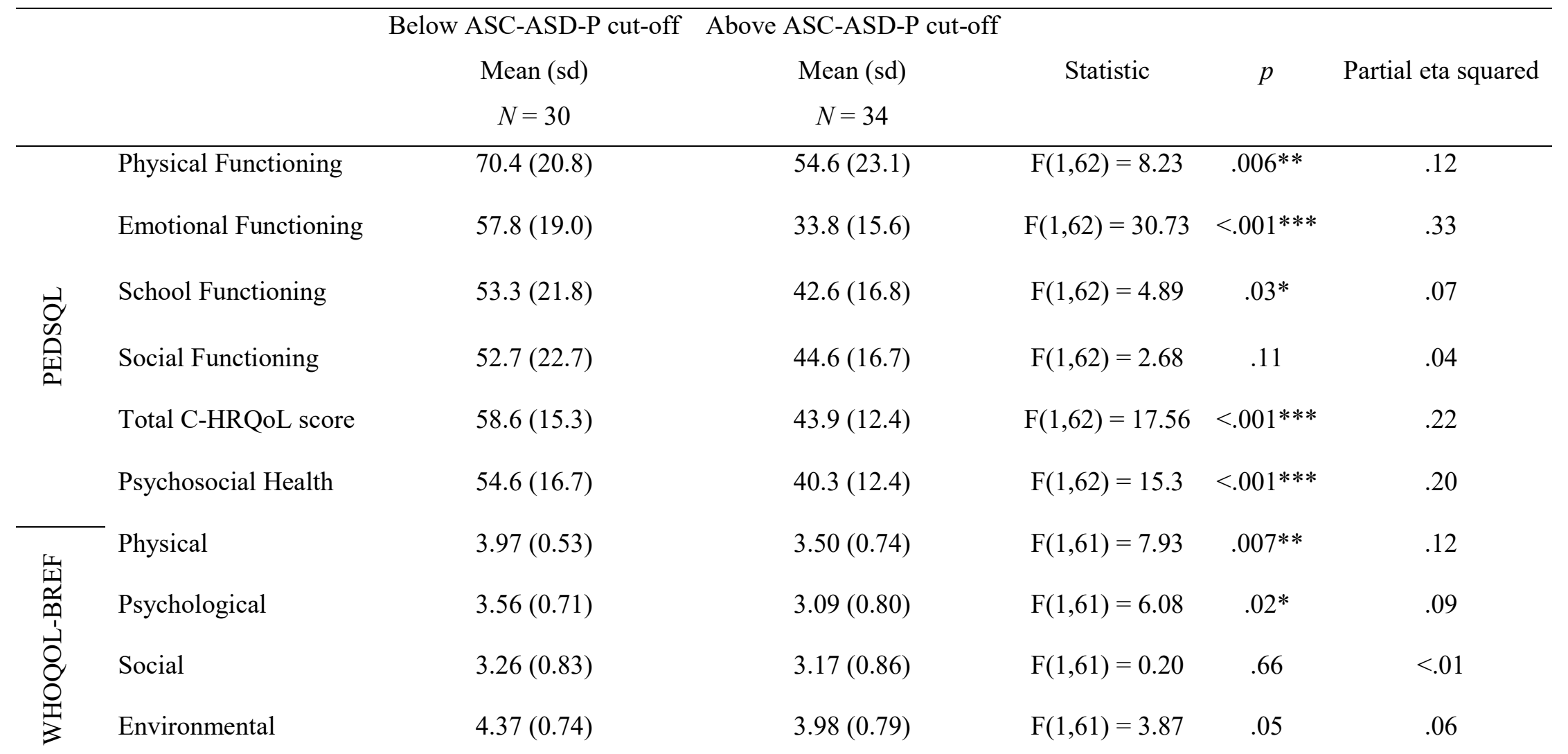


Total

$* p<.05 ; * * p<.01 * * * p<.001$.

$3.90(0.57)$

$3.50(0.66)$

$F(1,61)=6.41$

$.01 *$

.10 


\section{Influence of child anxiety symptomatology on c-HRQoL}

In order to explore whether specific aspects of a child's anxiety symptomatology predict overall and/or specific aspects of c-HRQoL, a series of linear regressions were undertaken. As autism characteristics have previously been shown to be predictive of c-HRQoL over and above child age and ability (de Vries \& Geurts, 2015), the child's total SCQ score was entered alongside the ASC-ASD-P subscale scores. The results are presented in Table 3 (n.b., higher scores on the c-HRQoL scale indicate a better quality of life, whilst higher scores on the ASC-ASD-P represent more anxiety symptomatology and higher scores on the SCQ represent more autism characteristics).

All models, with the exception of social functioning, were significant, predicting $18-46 \%$ of the variance. None of the ASC-ASD-P subscales or the SCQ total score were significant predictors of the overall c-HRQoL score; however, the following significant predictors were found: (1) total SCQ score $\left(\mathrm{R}^{2}=.26, p<.01\right)$ predicted physical functioning, with children with less autism characteristics having fewer difficulties with physical functioning; (2) ASC-ASD-P anxious arousal predicted the school functioning score $\left(\mathrm{R}^{2}=.16, p<.05\right)$, indicating that children with lower levels of anxious arousal have fewer difficulties with school functioning; and (3) ASC-ASD-P anxious arousal and separation anxiety scores predicted levels of emotional functioning $\left(\mathrm{R}^{2}=.46, p<.001\right)$ (see Table 3$)$. Standardised beta coefficients indicate that those with lower levels of separation anxiety and anxious arousal have fewer difficulties with emotional functioning. Hypotheses 1-3 can therefore all be rejected in relation to the total c-HRQoL score but each partially accepted for specific c-HRQoL domain scores. 


\section{Table 3.}

Results of Linear Regressions Exploring Anxiety (ASC-ASD-P) Subscales and Autism Characteristics (SCQ) Predicting c-HRQoL Subscales (PedsQL)

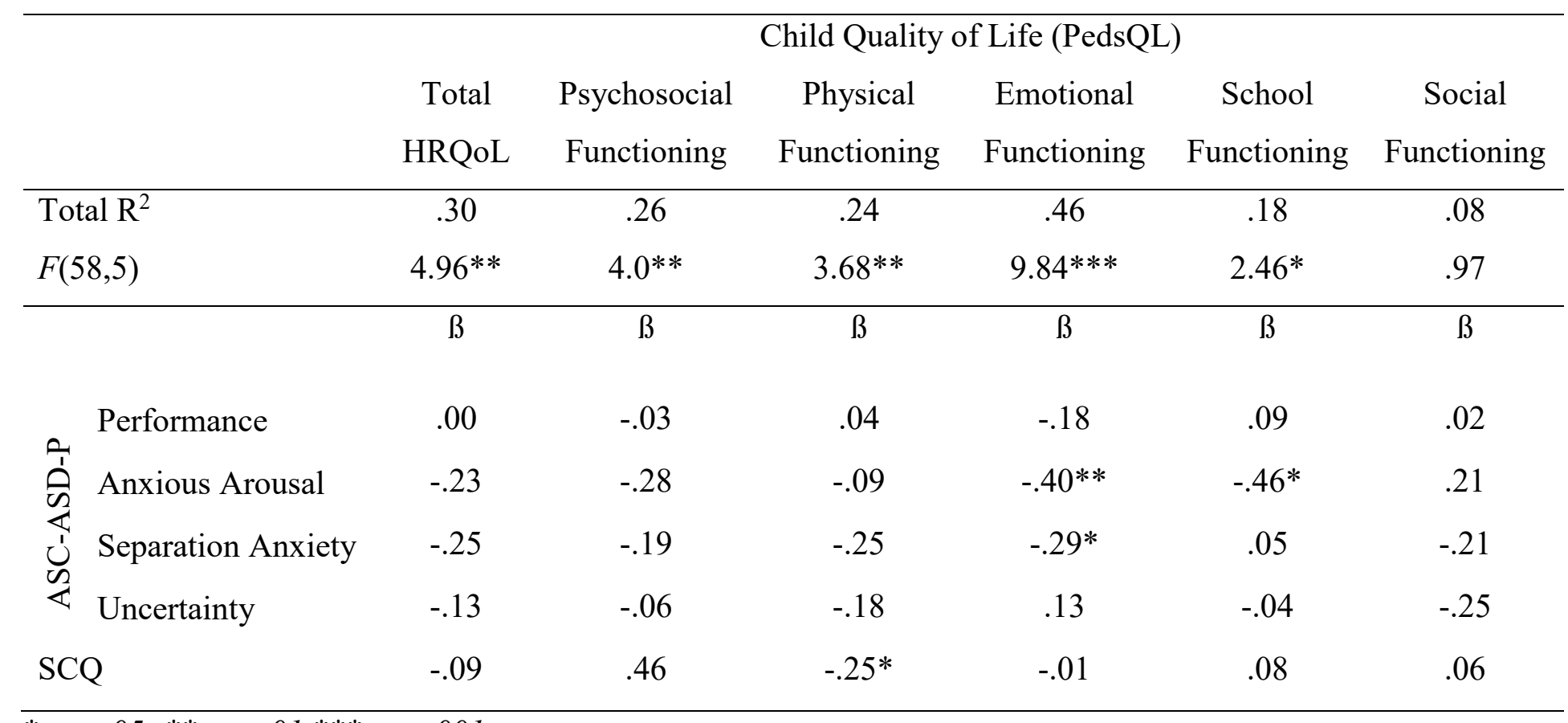

$* p<.05 ; * * p<.01 * * * p<.001$. 
Influence of child anxiety symptomatology on $p-Q o L$

In order to explore whether specific aspects of a child's anxiety symptomatology predict overall and/or specific aspects of p-QoL over and above autism characteristics, a series of linear regressions were undertaken. As shown in Table 4, none of the models were significant, only predicting $3-16 \%$ of the variance. 


\section{Table 4.}

Results of Linear Regressions Exploring Anxiety (ASC-ASD-P) Subscales and Autism Characteristics (SCQ) Predicting P-QoL (WHOQoL)

\begin{tabular}{|c|c|c|c|c|c|c|}
\hline & \multicolumn{5}{|c|}{ Parent Quality of Life (WHOQoL) } \\
\hline & & Total QoL & Physical Health & Psychological & Social Relationships & Environment \\
\hline \multicolumn{2}{|c|}{ Total $\mathrm{R}^{2}$} & .14 & .14 & .13 & .03 & .16 \\
\hline \multicolumn{2}{|c|}{$F(56,5)$} & 1.86 & 1.77 & 1.72 & .39 & 2.12 \\
\hline \multirow{5}{*}{ 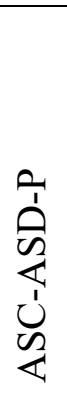 } & & $\beta$ & $\beta$ & $\beta$ & $\beta$ & $\beta$ \\
\hline & Performance & -.17 & -.28 & -.17 & -.11 & -.01 \\
\hline & Anxious Arousal & .36 & .22 & .35 & .24 & .37 \\
\hline & Separation Anxiety & -.30 & -.11 & -.34 & -.12 & $-.36^{*}$ \\
\hline & Uncertainty & -.18 & -.14 & -.11 & -.02 & -.27 \\
\hline \multicolumn{2}{|c|}{ SCQ } & -.07 & -.17 & .03 & .08 & -.09 \\
\hline
\end{tabular}

$* p<.05 ; * * p<.01 * * * p<.001$. 


\section{Discussion}

This is the first study to comprehensively explore whether anxiety and autism characteristics predict HRQoL of children on the autism spectrum and QoL in parents of children on the autism spectrum. The findings indicate that children on the spectrum with elevated anxiety experience significantly poorer overall c-HRQoL and have significantly poorer scores on the physical, emotional, and school functioning domains. In addition, their parents also experience overall poorer QoL, specifically in the physical health and psychological domains.

Through exploring the predictors of c-HRQoL in school-aged children on the spectrum, this study extends the previous literature by demonstrating that scores on specific subscales of the autism-specific anxiety measure, but not autism characteristics, predicted the emotional, social, and school functioning subscales of c-HRQoL. However, autism characteristics were the only significant predictor of the Physical Functioning subscale. Importantly, it was exploring the data firstly using total scores and then also with subscale/domain scores (on both the anxiety and c-HRQoL measure) that allowed for these relationships to be identified. While it has been established that children on the autism spectrum experience poorer QoL relative to typically developing children (van Heijst \& Geurts, 2015) and children with other co-occurring psychiatric and health conditions (Kose et al., 2013; Sikora et al., 2012), the range of factors that contribute to the c-HRQoL of children on the autism spectrum have been poorly understood. This study therefore builds upon the new and emerging literature proposing that psychiatric conditions such as anxiety have a negative impact on the c-HRQoL of children on the autism spectrum (de Vries \& Geurts, 2015; van Heijst \& Geurts, 2015; van Steensel et al., 2012). In contrast to the findings relating to C-HRQoL, anxiety symptomatology did not predict p-QoL. 


\section{Elevated anxiety and c-HRQoL and p-QoL}

There is a growing body of literature which suggests as well as experiencing "typical" anxiety, children on the spectrum may also experience anxiety differently from typically developing children (Kerns \& Kendall, 2012; White et al., 2015) and that measures designed for typically developing children may not capture the full range of anxiety symptoms experienced by children on the spectrum. This study was the first to explore c-HRQoL in children on the autism spectrum using an autism-specific measure of anxiety, the ASC-ASD$\mathrm{P}$, looking at both clinical cut-off scores and relative contributions of each subscale score. Using the elevated anxiety cut-off proposed by Rodgers et al. (n.d.), results revealed that children who experienced elevated levels of anxiety experienced poorer c-HRQoL overall (PedsQL total score) and psychosocial health as well as achieving lower scores in the physical, emotional, and school functioning domains, with all results having a medium to large effect size with the exception of school functioning, which had a small to medium effect size. This highlights that anxiety does not just impact children's emotional functioning, but that elevated anxiety symptomatology has a wide-ranging impact on children on the autism spectrum, which includes their physical and school functioning. This aligns with reports from individuals on the spectrum, their parents, and teachers (Saggers et al., 2016) which place anxiety in the top three factors affecting school participation and the top two factors influencing classroom support needs. The impact of elevated levels of anxiety also extends beyond the child, with parents of children with elevated anxiety reporting poorer total p-QoL and poorer physical health and psychological subscale scores of the WHO-QoL BREF. 
When controlling for autism characteristics as measured by the SCQ, ASC-ASD-P subscale scores were not found to significantly predict the any p-QoL scores nor the total cHRQoL or the Psychosocial Functioning score. However, when exploring specific domains of c-HRQoL through the subscale scores, it was found that $46 \%$ of the variance in emotional functioning scores could be explained by the ASC-ASD-P domains and SCQ score, with both anxious arousal and separation anxiety as significant predictors. One ASC-ASD-P subscale (anxious arousal) was a significant predictor of school functioning in the model which explained $18 \%$ of the variance. Children who were reported to experience higher anxious arousal symptoms experienced higher difficulties in the domain of emotional and school functioning. This may be somewhat explained by level of child ability, which was not measured in this study, although anxious arousal scores were not associated with child adaptive behaviour scores in previous research (den Houting et al., 2018b). It may be that heightened anxious arousal impacts upon a child's concentration, or may in itself be an aversive experience. Separation anxiety, identified as another leading anxiety subtype experienced by children on the autism spectrum (Simonoff et al., 2008), predicted the emotional functional subscale of c-HRQoL in the current study. Separation anxiety is problematic for children and the effects may extend beyond the time of separation. For example, the anticipatory worry associated with separating may contribute to sleep disturbances throughout the night which may then impact upon emotional and school functioning.

Interestingly, two subscales of the ASC-ASD-P did not predict any aspects of cHRQoL. Firstly, performance anxiety had a $\beta$ of $<.1$ for the total ASC-ASD-P score and three of the four subscales, including school functioning. Secondly, despite intolerance of uncertainty being identified as a significant factor in both anxiety and behavioural aspects of autism (Wigham et al., 2015), within this study, the Uncertainty subscale did not predict the 
c-HRQoL. A lack of relationship between difficulties with, or intolerance of, uncertainty and QoL has been reported in other populations, including individuals with epilepsy (Barahmand \& Haji, 2014) and women with breast cancer (Pahlevan Sharif, Ahadzadeh, \& Perdamen, 2017). Barahmand and Haji (2014), whose mediation models showed that irritability completely mediated the effects of both intolerance of uncertainty and worry on QoL, suggest that it may be the consequence of the intolerance of uncertainty (i.e., that it causes irritability and frustration) that reduces the c-HRQoL, rather than the difficulties with uncertainty per se. Further work is needed to explore and explain these results within the autism population as such mediation models have important implications for intervention focus (e.g., focus upon the mediator). These findings reiterate the importance of exploring specific aspects of a construct by going beyond the total scores; if only total scale scores were considered here, important information concerning the impact of specific subdomains of anxiety on specific subdomains of c-HRQoL would have been missed.

To date, some of the "core" features associated with autism (social communication difficulties), as well as behavioural problems, have been found to predict the c-HRQoL of children on the autism spectrum (Chiang \& Wineman, 2014). This has led to the suggestion that children on the autism spectrum experience poorer c-HRQoL that is attributed to the "severity" of their autism characteristics. Within this study, autism characteristics (as measured by the SCQ) predicted one (Physical functioning) domain of the PedsQL. However, when controlling for children's autism characteristics, anxiety symptoms were the strongest predictor of emotional and school functioning aspects of c-HRQoL, making a greater contribution over and above their autism characteristics. These are consistent with the work of Matson and Nebel-Schwalm (2007) who suggested that anxiety causes more disruption to the individual than their autism characteristics. Similarly, Renty and Roeyers (2006) also found that the QoL of adults on the autism spectrum was not related to their symptoms or the 
severity of their disability. These are important findings that may help to inform interventions and how outcomes are measured. It is worth noting that much of the early intervention literature measures cognitive and language outcomes, and considers a reduction in autism characteristics to be the "optimal outcome" (Fein et al., 2013; Starr, Propovic, \& McCall, 2016; Towle, Vacanti-Shova, Shristi, \& Higgins D'alessandro, 2014). However, in light of the current findings that convey the impact of anxiety on the c-HRQoL of children at school age, perhaps there should be a greater emphasis on identifying and targeting causes and triggers of children's anxiety as part of early childhood interventions to enhance c-HRQoL and potentially minimise later disruption at school age. Critically, the results from this study highlight the importance of developing and increasing access to effective treatments to help reduce anxiety in children on the autism spectrum as these in turn should enhance the child's QoL. Such targeted intervention would need to identify and (where possible) reduce the causes and/or triggers of the anxiety, including environmental factors, in order to reduce anxiety symptomatology experienced. The non-significant models exploring child anxiety symptomatology on p-QoL suggests that there may be a complex relationship which may be influenced by parent, family and social factors. It is likely that there may be mediating or moderating effects of coping strategies (Adams, Rose, Jackson, Karakstani \& Oliver, 2018; Benson, 2014) parenting behaviours (Osborne, McHugh, Saunders \& Reed, 2008), child age or ability (Adams et al., 2018) and systemic elements such as family income and demographics (Dardas \& Ahmad, 2014). There is a need for further studies, ideally using a large cohort and a longitudinal design, to explore and model a range of child, parent and family factors and their impact upon the relationship between child emotional and behavioural presentation and p-QoL (see meta-analysis by Yorke et al., 2018)

\section{Clinical implications}


The current findings have important implications for children's school adjustment. When controlling for autism characteristics, children's anxious arousal continued to have a significant impact on their school functioning over and above their autism characteristics. Children experiencing heightened anxious arousal at school may have more difficulty attending to and completing their schoolwork, which has the potential to impact their learning and subsequent development. Teachers need to be aware of the potential impact of a child's diagnosis when understanding their emotional experience and/or behavioural presentation (Oliver et al., 2013; Oliver, Woodcock, \& Adams, 2010). Teachers would benefit from tailored information to aid them in identifying the range of anxiety symptomatology in their students on the autism spectrum and therefore enable them to utilise strategies to support anxiety in the classroom $(<$ removed for blind review $>$ ) This could include a student's anxiety "signature"; an individualised profile of emotions, behaviours, expressions, verbalisations, or actions that indicate elevated anxiety for that child in that particular setting. Computerised systems which convey pupil-specific information about students on the autism spectrum to teachers through the lesson register, especially those which convey information created in dialogue with the student on the spectrum such as the FAMe ${ }^{\mathrm{TM}}$ approach described in Leatherland (2018), may open up the opportunities for sharing such information. A recent systematic review conducted by $<$ removed for blind review $>$ revealed a lack of current school-based anxiety and autism research, with the only available studies tending to report mean scores on measures rather than the actual profile of symptoms, limiting the extent to which current work is able to inform teachers of the key anxiety symptomatology and highlighting the need for further work in this area.

Heightened separation anxiety was found to impact on children's emotional functioning in the current study. This may impact upon social interactions with peers, an area that can be problematic for children on the autism spectrum who experience inherent social 
communication and peer interaction difficulties (Kasari, Locke, Gulsrud, \& Rotheram-Fuller, 2011; Macintosh \& Dissanayake, 2006). The results suggest that separation anxiety may benefit from being monitored in children on the autism spectrum from an early age in order to enhance c-HRQoL. Appropriate supports should be implemented as necessary to reduce any anxiety incurred by children when separating from their parents. Addressing early separation anxiety may prepare children for the separation incurred during the school transition, which may minimise the associated disruption and reduce the maladjustment at school.

\section{Limitations and future research directions}

The current study relied on parent report and, given that parents opted in to the study, may have a recruitment bias of parents interested in this area. Because the study collected all information via questionnaires, there was neither independent confirmation of autism diagnosis nor valid measure of child ability. The reliance upon parent report for c-HRQoL may be a limitation given the well-documented incongruence between child-self and proxyparent report (Clark, Magill-Evans, \& Koning, 2014; Ikeda, Hinckson, \& Krageloh, 2014). To that end, research has confirmed that children on the autism spectrum are able to provide valid subjective reports on their own well-being and functioning (Shipman, Sheldrick, \& Perrin, 2010). As such, a companion study gathering self-report data would complement the current findings, to inform how children on the autism spectrum perceive and report on their own anxiety and autism characteristics and how they consider that these factors impact on their c-HRQoL.

The implementation of the ASC-ASD-P assessment was a strength of this study as it allows consideration of autism-specific anxiety symptoms. However, the ASC-ASD-P would benefit from further evaluation in terms of both the areas covered as well as the validity and reliability against formal diagnostic interviews. A limitation was that the c-HRQoL measure 
was one designed for the typically developing population. Rodogno, Krause-Jensen, and Ashcroft (2016) discuss the shortcomings in current assessment and understanding of QoL for individuals on the autism spectrum which is founded on the philosophy of well-being in those without a diagnosis on the spectrum. The authors raise an important point such that we cannot and should not assume that well-being in individuals on the spectrum is the same as that for individuals not on the spectrum. Rodogno et al. (2016) argue the need for current assessments to be modified to be 'autism sensitive' to more accurately capture what it is that gives value to the lives of individuals on the autism spectrum. To that end, the recent development of a self-report c-HRQoL scale designed specifically for adults on the autism spectrum that has added questions relating to barriers to accessing services, friendships, sources of support, and sensory issues is welcome (McConachie et al., 2018), but this is yet to be conducted for children or young people. It is also important to be aware of the limitations of measurement via questionnaire without the opportunity to request clarification. For example, the SCQ would define repetitive behaviours as a characteristic of autism, whereas it may be that the repetitive behaviour is actually being used as a coping mechanism to reduce anxiety. As a coping strategy, repetitive behaviours may actually be adding to an individual's quality of life by reducing their anxiety, but when assumed as an autism characteristic, it is statistically associated with reducing quality of life. Additional examples of where further information would have been useful include improving the understanding around sample characteristics, for example, asking whether formal hearing assessments had been undertaken for the $9.9 \%$ who reported moderate to significant hearing difficulties or whether co-occurring diagnoses such as developmental coordination disorder may have been the cause of the child's difficulties with washing or dressing. Further work with careful measurement of such factors is required to further explore these issues. 
Finally, whilst the models and some of the predictors were significant, they do not account for all of the variance in c-HRQoL, meaning that further work with more predictors (and therefore a larger sample) is needed. A larger sample would allow for more factors, including more demographic factors, to be entered into the regression analysis. Future work should include a broader scope and measure environmental factors, including the sensory environment, and whether this interacts with the relationship between anxiety and quality of life in individuals on the autism spectrum.

\section{Conclusions}

To date, anxiety has been recognised as a significant mental health concern for children on the autism spectrum who are more vulnerable to experiencing anxiety at clinically significant levels when compared to typically developing peers (van Steensel et al., 2011; White et al., 2015). This is the first study to establish the contribution of anxiety on the c-HRQoL of school-aged children on the autism spectrum. Children experiencing elevated anxiety symptoms had significantly poorer total c-HRQoL, and specifically, significantly

poorer domains of emotional, school, and physical functioning. The current findings provide new insights in the c-HRQoL literature, as until now it has been widely suspected that it was the autism-related symptomatology that was limiting children's c-HRQoL. Instead, the current findings contribute a novel finding to the literature by identifying specific aspects of children's anxiety symptomatology as significant factors contributing to poorer functioning across a range of c-HRQoL domains.

\section{Ethical Approval}


All procedures performed in studies involving human participants were in accordance with the ethical standards of the institutional and/or national research committee and with the 1964 Helsinki declaration and its later amendments or comparable ethical standards.

\section{Informed Consent}

Informed consent was obtained from all individual participants included in the study.

\section{Compliance with Ethical Standards:}

This study was not funded by any funding body. The authors acknowledge the support of a small internal university grant to assist with data entry and management. 


\section{References}

Adams, D., Hastings, R., Alston-Knox, A., Cianfaglione, R., Eden, K., Felce, D., Griffith, G., Moss, J, Stinton, C. \& Oliver, C. (2018). Using Bayesian methodology to explore the profile of mental health and well-being in 646 mothers of children with 13 rare genetic syndromes in relation to mothers of children with autism. Orphanet Journal of Rare Diseases, 13, 185. doi: 10.1186/s13023-018-0924-1.

Adams, D., Rose, J. Jackson, N., Karakatsani, E., \& Oliver, C (2018). Coping strategies in mothers of children with intellectual disabilities showing multiple forms of challenging behaviour: associations with maternal mental health. Behavioural and Cognitive Psychotherapy. 46(3), 257-275. Doi: 10.1017/S1352465817000704.

Adams, D., Simpson, K., \& Keen, D. (2018). School-related anxiety symptomatology in a community sample of primary-school aged on the autism spectrum. Journal of School Psychology, 70, 64-73. Doi: 10.1016/j.jsp.2018.07.003.

Adams, D., Young, K., Simpson, K., \& Keen, D. (2018). Parent descriptions of the presentation and management of anxiousness in children on the autism spectrum. Autism, ePub ahead of Print. doi:10.1177/1362361318794031

Arias, V. B., Gomez, L. E., Moran, M. L., Alcedo, M. A., Monsalve, A., \& Fontanil, Y. (2018). Does quality of life differ for children with autism spectrum disorder and intellectual disability compared to peers without autism? Journal of Autism and Developmental Disorders, 48, 123-136. doi:10.1007/s10803-017-3289-8

Barahmand, U., \& Haji, A. (2014). The impact of intolerance of uncertainty, worry and irritability on quality of life in persons with epilepsy: Irritability as mediator. Epilepsy Research, 108, 1335-1344. doi:10.1016/j.eplepsyres.2014.07.002 
Beesdo, K., Knappe, S., \& Pine, D. S. (2009). Anxiety and anxiety disorders in children and adolescents: Developmental issues and implications for DSM-V. Psychiatric Clinics of North America, 32, 483-524. doi:10.1016/j.psc.2009.06.002

Benson, P.R. (2014) Coping and psychological adjustment among mothers of children with ASD: An accelerated longitudinal study. Journal of Autism and Developmental Disorders, 44, 1793-1807

Biggs, E. E., \& Carter, E. W. (2016). Quality of life for transition-age youth with autism or intellectual disability. Journal of Autism and Developmental Disorders, 46, 190-204. doi:10.1007/s10803-015-2563-x

Birmaher, B., Brent, D., Chiapetta, L., Bridge, J., Monga, S., \& Baugher, M. (1999). Psychometric properties of the screen for child anxiety related emotional disorders (SCARED): A replication study. Journal of the American Academy of Child \& Adolescent Psychiatry, 38, 1230-1236.

Bittner, A., Egger, H. L., Erkanli, A., Jane Costello, E., Foley, D. L., \& Angold, A. (2007). What do childhood anxiety disorders predict? Journal of Child Psychology and Psychiatry, 48, 1174-1183. doi:10.1111/j.1469-7610.2007.01812.x

Bourke-Taylor, H., Pallant, J. Law, M. \& Howlie, L., (2012). Predicting mental health among mothers of school-aged children with developmental disabilities: the relative contribution of child, maternal and environmental factors. Res Dev Disabil, 33, 173240. doi: 10.1016/j.ridd.2012.04.011

Caporino, N. E., Read, K. L., Shiffrin, N., Settipani, C., Kendall, P. C., Compton, S. N., .. . Albano, A. M. (2017). Sleep-related problems and the effects of anxiety treatment in children and adolescents. Journal of Clinical Child and Adolescent Psychology, 46(5), 675-685. doi:10.1080/15374416.2015.1063429 
Centers for Disease Control and Prevention. (2018). Prevalence of autism spectrum disorders among children aged 8 years - autism and developmental disabilities monitoring network, 11 sites, United States, 2014. Morbidity and Mortality Weekly Report. Surveillance Summaries, 67, 1-23.

Chesnut, S., Wei, T., Barnard-Brak, L., \& Richman, D. (2017). A meta-analysis of the social communication questionnaire: Screening for autism spectrum disorder. Autism, 21, 920-928. doi:10.1177/1362361316660065

Chiang, H.-M., \& Wineman, I. (2014). Factors associated with quality of life in individuals with autism spectrum disorders: A review of literature. Research in Autism Spectrum Disorders, 8, 974-986. doi:10.1016/j.rasd.2014.05.003

Chorpita, B. F., Yim, L., Moffitt, C., Umemoto, L. A., \& Francis, S. E. (2000). Assessment of symptoms of DSM-IV anxiety and depression in children: A revised child anxiety and depression scale. Behaviour Research and Therapy, 38, 835-855.

Clark, B. G., Magill-Evans, J. E., \& Koning, C. J. (2014). Youth with autism spectrum disorders. Focus on Autism and Other Developmental Disabilities, 30(1), 57-64. doi:10.1177/1088357614522289

Dardash, L.A., \& Ahmad, M.M. (2014). Predictors of quality of life for fathers and mothers of children with autistic disorder. Research in Developmental Disabilities, 35, 13261333. doi: 10.1016/j.ridd.2014.03.009

den Houting, J., Adams, D., Roberts, J., \& Keen, D. (2018a). Exploring anxiety symptomatology in school-aged autistic children using an autism-specific assessment. Research in Autism Spectrum Disorders, 50, 73-82. doi: 10.1016/j.rasd.2018.03.005. den Houting, J., Adams, D., Roberts, J. \& Keen, D (2018b). An exploration of autismspecific and non-autism-specific measures of anxiety symptomatology in school-aged autistic children. Clinical Psychologist, ePub ahead of print. doi: 10.1111/cp.12174 
de Vries, M., \& Geurts, H. (2015). Influence of autism traits and executive functioning on quality of life in children with an autism spectrum disorder. Journal of Autism and Developmental Disorders, 45, 2734-2743. doi:10.1007/s10803-015-2438-1

Egilson, S. T., Olafsdottir, L. B., Leosdottir, T., \& Saemundsen, E. (2017). Quality of life of high-functioning children and youth with autism spectrum disorder and typically developing peers: Self- and proxy-reports. Autism, 21, 133-141. doi:10.1177/1362361316630881

Fein, D., Barton, M., Eigsti, I. M., Kelley, E., Naigles, L., Schultz, R. T., . . Tyson, K. (2013). Optimal outcome in individuals with a history of autism. Journal of Child Psychology and Psychiatry, 54, 195-205. doi:10.1111/jcpp.12037

Gilbertson, T. J., Morgan, A. J., Rapee, R. M., Lyneham, H. J., \& Bayer, J. K. (2017). Psychometric properties of the Child Anxiety Life Interference Scale - Preschool Version. Journal of Anxiety Disorders, 52, 62-71. doi:10.1016/j.janxdis.2017.10.002

Gregory, A. M., Buysse, D. J., Willis, T. A., Rijsdijk, F. V., Maughan, B., Rowe, R., .. . Eley, T. C. (2011). Associations between sleep quality and anxiety and depression symptoms in a sample of young adult twins and siblings. Journal of Psychosomatic Research, 71, 250-255. doi:10.1016/j.jpsychores.2011.03.011

Gregory, A. M., \& Sadeh, A. (2012). Sleep, emotional and behavioral difficulties in children and adolescents. Sleep Medicine Reviews, 16(2), 129-136. doi:10.1016/j.smrv.2011.03.007

Ivanenko, A., McLaughlin Crabtree, V., O’Brien, L. \& Gozal, D. (2006). Sleep complaints and psychiatric symptoms in children evaluated at a pediatric mental health clinic. Journal of Clinical Sleep Medicine, 2(1), 42-48. 
Ikeda, E., Hinckson, E., \& Krageloh, C. (2014). Assessment of quality of life in children and youth with autism spectrum disorder: A critical review. Quality of Life Research, 23(4), 1069-1085. doi:10.1007/s11136-013-0591-6

Kasari, C., Locke, J., Gulsrud, A., \& Rotheram-Fuller, E. (2011). Social networks and friendships at school: Comparing children with and without ASD. Journal of Autism and Developmental Disorders, 41, 533-544. doi:10.1007/s10803-010-1076-X

Keen, D., Adams, D., Simpson, K., den Houting, J., \& Roberts, J. (2017). Anxiety-related symptomatology in young children on the autism spectrum. Autism, ePub ahead of print. doi:10.1177/1362361317734692

Kerns, C., \& Kendall, P. C. (2012). The presentation and classification of anxiety in autism spectrum disorder. Clinical Psychology Science and Practice, 19, 323-347.

Kingery, J. N., Erdley, C. A., Marshall, K. C., Whitaker, K. G., \& Reuter, T. R. (2010). Peer experiences of anxious and socially withdrawn youth: An integrative review of the developmental and clinical literature. Clinical Child and Family Psychology Review, 13(1), 91-128. doi:10.1007/s10567-009-0063-2

Kose, S., Erermis, S., Ozturk, O., Ozbaran, B., Demiral, N., Bildik, T., \& Aydin, C. (2013). Health related quality of life in children with autism spectrum disorders: The clinical and demographic related factors in Turkey. Research in Autism Spectrum Disorders, 7(2), 213-220. doi:10.1016/j.rasd.2012.09.009

Kuhlthau, K., Orlich, F., Hall, T., Sikora, D. M., Kovacs, E., Delahaye, J., \& Clemons, T. (2010). Health related quality of life in children with autism spectrum disorders: Results from the Autism Treatment Network. Journal of Autism and Developmental Disorders, 40, 721-729.

Lecavalier, L., Wood, J. J., Halladay, A. K., Jones, N. E., Aman, M. G., Cook, E. H., . . Scahill, L. (2014). Measuring anxiety as a treatment endpoint in youth with autism 
spectrum disorder. Journal of Autism and Developmental Disorders, 44, 1128-1143. doi:10.1007/s10803-013-1974-9

Leatherland, J. (2018). Understanding how autistic pupils experience secondary school: autism criteria, theory and FAMe ${ }^{\mathrm{TM}}$ (Doctoral dissertation, Sheffield Hallam University).

Lidstone, J., Uljarević, M., Sullivan, J., Rodgers, J., McConachie, H., Freeston, M., . . . Leekam, S. (2014). Relations among restricted and repetitive behaviors, anxiety and sensory features in children with autism spectrum disorders. Research in Autism Spectrum Disorders, 8(2), 82-92. doi:10.1016/j.rasd.2013.10.001

Limbers, C. A., Heffer, R. W., \& Varni, J. W. (2009). Health-related quality of life and cognitive functioning from the perspective of parents of school-aged children with Asperger's syndrome utilizing the PedsQL ${ }^{\mathrm{TM}}$. Journal of Autism and Developmental Disorders, 39, 1529-1541. doi:10.1007/s10803-009-0777-5

Macintosh, K., \& Dissanayake, C. (2006). A comparative study of the spontaneous social interactions of children with high-functioning autism and children with Asperger's disorder. Autism, 10, 199-220. doi:10.1177/1362361306062026

Mahrer, N. E., Montano, Z., \& Gold, J. I. (2012). Relations between anxiety sensitivity, somatization, and health-related quality of life in children with chronic pain. $J$ Pediatric Psychology, 37, 808-816. doi:10.1093/jpepsy/jss054

Matson, J. L., \& Nebel-Schwalm, M. S. (2007). Comorbid psychopathology with autism spectrum disorder in children: An overview. Research in Developmental Disabilities, 28, 341-352. doi:10.1016/j.ridd.2005.12.004

McConachie, H., Mason, D., Parr, J. R., Garland, D., Wilson, C., \& Rodgers, J. (2018). Enhancing the validity of a quality of life measure for autistic people. Journal of 
Autism and Developmental Disorders, 48, 1596-1611. doi:10.1007/s10803-017-3402-

$\mathrm{Z}$

Oliver, C., Adams, D., Allen, D., Bull, L., Heald, M., Moss, J., . . Woodcock, K. (2013). Causal models of clinically significant behaviors in Angelman, Cornelia de Lange, Prader-Willi and Smith-Magenis syndromes. In R. Hastings \& J. Rojahn (Eds.), Challenging behavior (Vol. 44, pp. 167-211). London: Elsevier.

Oliver, C., Woodcock, K., \& Adams, D. (2010). The importance of aetiology for intellectual disability. In G. Grant, P. Ramcharan, M. Flynn, \& M. Richardson (Eds.), Learning disability: A life cycle approach to valuing people (pp. 135-146).Open University Press/Wiley.

Osborne L.A., McHugh L., Saunders J., Reed P. (2008). The effect of parenting behaviors on subsequent child behavior problems in autistic spectrum conditions. Research in Autism Spectrum Disorders, 2, 249-263.

Pahlevan Sharif, S., Ahadzadeh, A. S., \& Perdamen, H. K. (2017). Uncertainty and quality of life of Malaysian women with breast cancer: Mediating role of coping styles and mood states. Applied Nursing Research, 38, 88-94. doi:10.1016/j.apnr.2017.09.012

Renty, J. O., \& Roeyers, H. (2006). Quality of life in high-functioning adults with autism spectrum disorder: The predictive value of disability and support characteristics. Autism, 10(5), 511-524. doi:10.1177/1362361306066604

Rodgers, J., Hodgson, A., Shields, K., Wright, C., Honey, E., \& Freeston, M. (2017). Towards a treatment for intolerance of uncertainty in young people with autism spectrum disorder: Development of the Coping with Uncertainty in Everyday Situations (CUES(c)) Programme. Journal of Autism and Developmental Disorders, 47, 3959-3966. doi:10.1007/s10803-016-2924-0 
Rodgers, J., Wigham, S., McConachie, H., Freeston, M., Honey, E., \& Parr, J. R. (2016). Development of the anxiety scale for children with autism spectrum disorder (ASCASD). Autism Research, 9, 1205-1215. doi:10.1002/aur.1603

Rodgers J., Wigham S., McConachie H., et al. (nd) Anxiety Scale for Children - Autism Spectrum Disorder (ASC-ASD): Guidelines for use.

Rodogno, R., Krause-Jensen, K., \& Ashcroft, R. E. (2016). 'Autism and the good life': a new approach to the study of well-being. J Med Ethics, 42, 401-408. doi:10.1136/medethics-2016-103595

Rutter, M., Bailey, A., Lord, C., \& Berument, S. K. (2003). Social Communication Questionnaire. Los Angeles, CA: Western Psychological Services.

Saggers, B., Klug, D., Harper-Hill, K., Costley, D., Clark, T., Bruck, S., . . Carrington, S. (2016). Australian autism educational needs analysis - What are the needs of schools, parents and students on the autism spectrum? Brisbane: Cooperative Research Centre for Living with Autism.

Shipman, D., Sheldrick, C., \& Perrin, E. (2010). Quality of life in adolescents with autism spectrum disorders: Reliability and validity of self reports. Journal of Developmental and Behavioral Pediatrics, 32(2), 85-89.

Spence, S., Donovan, C., \& Brechman-Toussaint, M. (1999). Social skills, social outcomes, and cognitive features of childhood social phobia. Journal of Abnormal Psychology, 108(2), 211-221.

Starr, E., Propovic, S., \& McCall, B. (2016). Supporting children with autism spectrum disorder at primary school: Are the promises of early intervention maintained? Current Developmental Disorder Review, 3, 46-56. doi:10.1007/s40474-016-0069-7

Stokes, M., Kornienko, L., Scheeren, A., Koot, H. M., \& Begeer, S. (2017). A comparison of children and adolescent's self-report and parental report of the PedsQL among those 
with and without autism spectrum disorder. Quality of Life Research, 26, 611-624. doi:10.1007/s11136-016-1490-4

Tavernor, L., Barron, E., Rodgers, J., \& McConachie, H. (2013). Finding out what matters: Validity of quality of life measurement in young people with ASD. Child: Care, Health and Development, 39, 592-601. doi:10.1111/j.1365-2214.2012.01377.x

The WHOQOL Group. (1998). The World Health Organization quality of life assessment (WHOQOL): Development and general psychometric properties. Social Sciences and Medicine, 46, 1569-1585.

Thomas, S., Sciberras, E., Lycett, K., Papadopolous, N., \& Rinehart, N. (2018). Physical functioning, emotional, and behavioral problems in children with ADHD and comorbid ASD: A cross-sectional study. Journal of Attention Disorders, 22(10), 1002-1007. doi:10.1177/1087054715587096

Totsika, V., Hastings, R.P., Emerson, E., Berridge, D. \& Lancaster, G. (2011). Behavior Problems at 5 Years of Age and Maternal Mental Health in Autism and Intellectual Disability. J Abnorm Child Psychol, 39, 1137-1147. https://doi.org/10.1007/s10802$011-9534-2$

Totsika, V., Felce, D., Kerr, M., \& Hastings, R. P. (2010). Behavior problems, psychiatric symptoms, and quality of life for older adults with intellectual disability with and without autism. Journal of Autism and Developmental Disorders, 40(10), 1171-1178. doi:10.1007/s10803-010-0975-1

Towle, P., Vacanti-Shova, K., Shristi, S., \& Higgins D'alessandro, A. (2014). School-age functioning of children diagnosed with autism spectrum disorder before age three: Parent reported diagnostic, adaptive, medication and school placement outcomes. Journal of Autism and Developmental Disorders, 44, 1357-1372. doi:10.1007/s10803-013-1997-2 
Tung, L.-C., Huang, C.-Y., Tseng, M.-H., Yen, H.-C., Tsai, Y.-P., Lin, Y.-C., \& Chen, K.-L. (2014). Correlates of health-related quality of life and the perception of its importance in caregivers of children with autism. Research in Autism Spectrum Disorders, 8, 1235-1242. doi:10.1016/j.rasd.2014.06.010

van Steensel, F. J., Bogels, S. M., \& Perrin, S. (2011). Anxiety disorders in children and adolescents with autistic spectrum disorders: A meta-analysis. Clinical Child and Family Psychology Review, 14, 302-317. doi:10.1007/s10567-011-0097-0

van Steensel, F. J., Bogels, S. M., \& Dirksen, C.D.. (2012). Anxiety and quality of life: clinically anxious children with and without autism spectrum disorders compared. Journal Clinical Child and Adolescent Psychology, 41, 731-8. doi: $10.1080 / 15374416.2012 .698725$

Varni, J. W., Seid, M. \& Kurtin, P. S. (2001). The Pedsql 4.0: Reliability and validity of the Pediatric Quality of Life Inventory, Version 4.0. Generic core scales in healthy and patient populations. Medical Care, 39, 800-812.

Vasilopoulou, E., \& Nisbet, J. (2016). The quality of life of parents of children with autism spectrum disorder: A systematic review. Research in Autism Spectrum Disorders, 23, 36-49. doi: 10.1016/j.rasd.2015.11.008

White, S., Lerner, M., McLeod, B., Wood, J. J., Ginsburg, G., Kerns, C., . . Compton, S. N. (2015). Anxiety in youth with and without autism spectrum disorder: Examination of factorial equivalence. Behavior Therapy, 46, 40-53.

Wigham, S., Rodgers, J., South, M., McConachie, H., \& Freeston, M. (2015). The interplay between sensory processing abnormalities, intolerance of uncertainty, anxiety and restricted and repetitive behaviours in autism spectrum disorder. Journal of Autism and Developmental Disorders, 45, 943-952. doi:10.1007/s10803-014-2248-x 
Yorke, I., White, P., Weston, A., Rafla, M., Charman, T., \& Simonoff, E. (2018). The Association Between Emotional and Behavioral Problems in Children with Autism Spectrum Disorder and Psychological Distress in Their Parents: A Systematic Review and Meta-analysis. Journal of autism and developmental disorders, 48, 3393-3415.

Rodogno, R., Krause-Jensen, K., \& Ashcroft, R. E. (2016). 'Autism and the good life': a new approach to the study of well-being. J Med Ethics, 42(6), 401-408.

doi:10.1136/medethics-2016-103595 